\title{
Solitary Intramuscular Cysticercosis, a Case Report from 47-year-old Man
}

\author{
Rasheed Mumini Wemimo ${ }^{1}$, Afolayan Enoch Abiodun ${ }^{1}$, Balogun Musbau Olusesan ${ }^{2}$, \\ Folaranmi Olaleke Oluwasegun ${ }^{2}$, Abdullahi Kabiru ${ }^{3}$, Shuaibu Usman Yahaya ${ }^{4}$, Mohammed Umar ${ }^{3}$, \\ Odebiyi Hassan Abiola ${ }^{5}$
}

${ }^{1}$ Department of Histopathology, Leah Medical Centre, Ilorin, Kwara State, Nigeria

${ }^{2}$ Department of Histopathology, University of Ilorin Teaching Hospital, Ilorin, Kwara State, Nigeria

${ }^{3}$ Department of Histopathology, Usmanu Danfodiyo University Teaching Hospital, Sokoto, Nigeria

${ }^{4}$ Department of Medical Microbiology, Federal Medical Centre, Birnin-Kudu, Jigawa State, Nigeria

${ }^{5}$ Department of Haematology, Federal Medical Centre, Birnin-Kudu, Jigawa State, Nigeria

\section{Email address:}

muminirasheed265@gmail.com (R. M. Wemimo)

\section{To cite this article:}

Rasheed Mumini Wemimo, Afolayan Enoch Abiodun, Balogun Musbau Olusesan, Folaranmi Olaleke Oluwasegun, Abdullahi Kabiru, Shuaibu Usman Yahaya, Mohammed Umar, Odebiyi Hassan Abiola. Solitary Intramuscular Cysticercosis, a Case Report from 47-year-old Man. International Journal of Infectious Diseases and Therapy. Vol. 5, No. 3, 2020, pp. 45-47. doi: 10.11648/j.ijidt.20200503.11

Received: June 13, 2020; Accepted: June 28, 2020; Published: July 13, 2020

\begin{abstract}
Cysticercosis is a common parasitic infection in developing countries involving the central nervous system (CNS), adnexal structures of the eye, skeletal muscle, and subcutaneous tissue. The principal mechanism of transmission is through ingestion of Taenia Solium eggs or contamination of fruits and vegetables fertilized with contaminated faecal materials. The eggs hatch within the small intestine and larvae travel to through blood vessels to the subcutaneous tissue, muscle, CNS, eye and other tissues where they eventually form cyst with host inflammatory response. The clinical features are variable from painful or painless swelling in the subcutaneous tissue to neurocysticercosis comprising of symptomatic headache, seizures and focal neurologic deficit. Solitary intramuscular cysticercosis without CNS involvement have also been reported, although it is rare. Thus, we present a case of solitary intramuscular cysticercosis involving bicep muscle in a 47-year-old engineer, a rare site without any neurologic or systemic manifestation.
\end{abstract}

Keywords: Cysticercosis, Bicep Muscle, MRI, and CT Scan

\section{Introduction}

Cysticercosis is a parasitic infection caused by encysted larvae of Taenia solium, the pork tapeworm. [1] It occurs worldwide and is the most common human parasitic infection of the nervous system in immunocompetent individuals. It has spread worldwide owing to travel from endemic zone in developing and few developed countries like Mexico, Central and South America, Africa, India, China, Eastern Europe, and Indonesia. [2, 3] Tapeworm infections are common in developing countries where there is poor access to sanitation facilities and close interaction between humans and animals, especially where pigs are used as a major source of meat or oil production. Cysticercosis is acquired through is through faeco-oral transmission caused by ingestion of eggs that are shed in the faeces of a human carrier. This can occur via infected food handlers that do not observe good personal hygiene or through consumption of fruits and vegetables fertilized by contaminated human waste. [ 1 The outer wall of the cyst is digested by gastric secretion to release oncospheres which contains suckers and hooks. The hooks are used for anchorage to the intestinal mucosa while suckers proliferate and mature in order to develop into an adult tapeworm. The adult tapeworm sheds eggs and proglottids that serve as source of infection for humans and pigs. The hatched oncospheres invade the intestinal wall, enter the mesenteric venules and spread into tissues and organs where they subsequently develop into cysts. They can lodge in any part of the body but the most common sites are the brain, eye and skeletal muscles. [4,5]. Cysticercosis infestation have 
been reported in varying organs such as adnexal structures of the eye, upper lip, brain, skeletal and muscle. [6-10]. The encysted larval form of $T$. Solium is otherwise known as cysticercus cellulosae. This larval stage can remain viable for many years in humans and the number of the cysts vary depending on burden of the disease. [5] Immune recognition are evaded without inflammatory response and are usually asymptomatic during this stage except for local swelling at the site of lodgement. [5] Acute inflammatory response is usually triggered by the death of the larva which might present as myalgia with chronic inflammation around the cyst through intermittent leakage of the intracystic fluid. Cysticercosis can affect any age or sex and clinical presentation is variable depend on disease burden, anatomic location and associated inflammation. [11, 12] The brain and eyes cyst are the most common sites associated with significant morbidity, accounting for $60-90 \%$ and $1-3 \%$ respectively. [11] The most plausible reason for the rapid growth of the cysticercus larval in the brain, eye and skeletal muscles can be attributed to the high blood flow compared to other organs. Isolated muscle involvement by cysticercosis is rare and is difficult to diagnose due to nonspecific clinical presentation mimicking a variety of other diseases such as abscess and tumour. [6] In the muscular form, three types of clinical manifestations have been described: the myalgic type, mass-like or pseudotumour and the rare pseudohypertrophic type, depending upon the pathogenesis as described above. [13] Therefore central nervous system or ocular involvement should always be ruled out with history, examination and investigations. [11] This case report is aimed at documentation of isolated intramuscular cysticercosis without central nervous system involvement in Ilorin at the Department of histopathology, Leah Medical Centre, Kwara State, Nigeria.

\section{A Case Report}

A 47-year-old male patient presented to the surgical outpatient department (OPD) in a private health facility with a painful swelling that had been gradually increasing in size over the left bicep muscle region for a duration of 3 months. He was an engineer who had been taking regular diet comprised of beef, vegetables but had no history of pork consumption. There was no history of headache, dizziness, blurring of vision and swelling in any other parts of the body. On examination, an ill-defined deep-seated mass was felt deep to the bicep muscle and measures $4.0 \times 4.0 \mathrm{~cm}$. The swelling was cystic in nature, freely movable, and tender. The overlying skin was normal. There was no neurological deficit or systemic abnormalities elicited. Investigation done were: Full blood counts: PCV 37\% (Normal), White blood cell counts $6.5 \times 10^{9} / \mathrm{L}$, (Normal), Platelet $150.0 \times 10^{9} / \mathrm{L}$ (Normal), MCV: 89fl (Normal); MCH: 32pg (Normal); and MCHC: 34dl/L (Normal). Urinalysis, Electrolyte/Urea/Creatinine and Fasting blood sugar were within normal reference interval. Radiological investigations comprising of Ultrasound, Magnetic Resonance Imaging
(MRI) and Computed Tomography (CT) were not done at the referring private health facility. The cyst was surgically excised en bloc with minimal blood loss. The surgical diagnosis was soft tissue tumour with query cause. The excised cyst was sent for histopathological analysis at the Department of Histopathology, Leah Medical Centre, Ilorin, Kwara State. The specimen consisted of 2 pieces of ruptured cystic specimens of about equal sizes and each measures $2.3 \mathrm{~cm} \times 2.0 \mathrm{~cm}$. The outer and inner wall of the cyst was smooth and glistening. Sections were submitted into tissue cassettes and was subsequently fixed, dehydrated in graded concentrations of alcohol, cleared in xylene and infiltrated with molten paraffin wax. The tissues were embedded and thin sections were cut at $3.0 \mathrm{um}$ and stained with haematoxylin and eosin. The histology diagnosis was reported as cysticercosis. The surgeon was delighted with the diagnosis and commenced patient on praziquantel (50 $\mathrm{mg} / \mathrm{kg} /$ day).

Micrograph

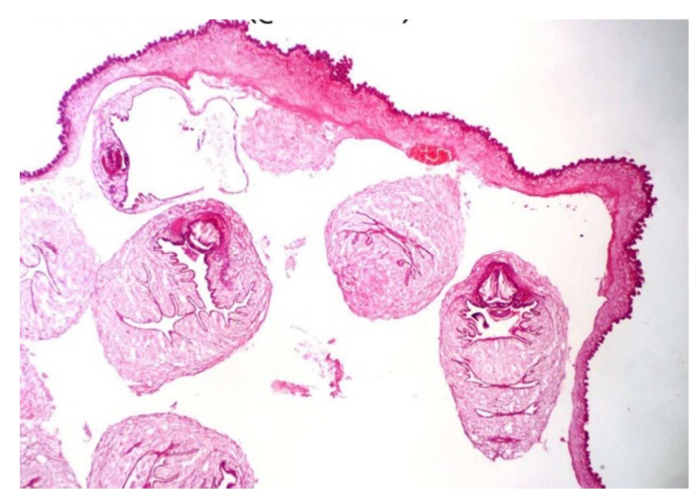

Figure 1. $x 100$ mag. Sections show wall of a cyst composed of fibrocollagenous tissue with the larval form containing scolex and hooklets.

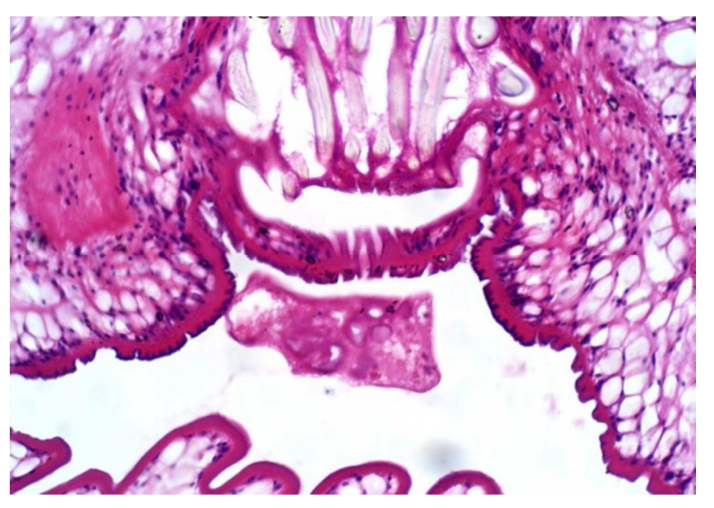

Figure 2. $x$ 400mag. Sections show larval form containing scolex and hooklets. There are duct-like invagination, double layer with eosinophilic substance.

\section{Discussion}

Human cysticercosis is an infection caused by the larval stage (cysticercus) of Taenia solium. It is commonly seen in developing countries due to overcrowding, poor sanitation, and unhygienic conditions, allowing greater contact between humans and pigs. This increases the chances of faecal contamination of food and water. [8] The larva develops into 
an adult worm in the intestinal mucosa within 5-12 weeks. The eggs and proglottids of the Taenia solium are passed in the faeces, and these serve as the source of infection for pigs and humans. Oncospheres from the ingested egg released in the presence of gastric secretions penetrate the intestinal wall to enter the mesenteric venules and subsequently reaching various sites of the body. This index isolated intramuscular case report is similar to findings by other authors. [14-16] The intramuscular cysticercosis may present clinically as myalgia, pseudotumor, soft tissue tumour, lipomas, epidermoid cysts, granular cell tumours, neuroma, neurofibromas, sarcoma, myxoma and pyomyositis. [13]. In this index case, the possibly source of infection was verified with no history of headache, seizure, blurring of vision, swelling in any other part of the body. However, there was history of eating meat and vegetable at different working sites and several restaurants while discharging his duties. This history of eating out at several eatery outlets is taken as possible source of infection from infected food handler or vegetable contaminated with human faeces containing the Taenia $S$ eggs. Although, stool microscopy for detection of parasite eggs was not done because the surgeon did not entertain a clinical diagnosis of cysticercosis as the time of evaluating the patient. Clinical diagnosis of solitary cysticercosis is difficult owing to the lack of other systemic manifestations. Thus, a definitive diagnosis is of cysticercosis can only be made when an excised specimen is submitted for histopathological diagnosis. It is germane to submit all excised surgical specimens for histopathology analysis and this will ensure timely diagnosis and direct the treatment options available for the patients.

\section{Conclusion}

Isolated intramuscular cysticercosis should be kept in mind as a differential diagnosis of any swelling or pseudo-mass of uncertain aetiology. The lesion can be reliably diagnosed with ultrasound scan and excision for definitive histopathological diagnosis with subsequent commencement of patients on anti-helminthics. Without histological analysis of this index case from a private hospital, the case would have been missed. Thus, this will contribute to underdiagnosis or under-reporting of cysticercosis.

\section{References}

[1] Meena D, Gupta M, Jain VK, Arya RK. Isolated intramuscular cysticercosis: Clinicopathological features, diagnosis and management - A review. J Clin Orthop Trauma. 2016; 7 (2): 243-249.
[2] Kraft R. Cysticercosis: an emerging parasitic disease. Am Fam Physician. 2007; 76 (1): 91-96.

[3] Garcia H. H., Del Brutto O. H. Taenia solium cysticercosis. Infect Dis Clin N Am. 2000; 14 (1): 97-119.

[4] Yamashita P., Kelsey J., Henderson S. O. Subcutaneous cysticercosis. J Emerg Med. 1998; 16 (July-August (4)): 583586.

[5] Mittal A., Das D., Iyer N., Nagaraj J., Gupta M. Masseter cysticercosis - a rare case diagnosed on ultrasound. Dentomaxillofac Radiol. 2008; 37 (2): 113-116.

[6] Abdelwahab I. F., Klein M. J., Hermann G., Abdul-Quader M. Solitary cysticercosis of the biceps brachii in a vegetarian: a rare and unusual pseudotumor. Skelet Radiol. 2003; 32 (7)): 424-428.

[7] Kaliaperumal S, Rao V A, Parija S C. Cysticercosis of the eye in south India - A case series. Indian J Med Microbiol 2005; 23: $227-30$.

[8] Deshmukh A, Avadhani A, Tupkari J V, Sardar M. Cysticercosis of the upper lip. J Oral Maxillofac Pathol 2011; 15: 219-222.

[9] Syed A. A., Ayman M. S, Hanns F, Hussain M. A, Jasmin A, Ronda D. E et al., Neurocysticercosis: A case report and brief review. Asian Pacific Journal of Tropical Medicine 2016; 9 (1): 100-102.

[10] Purohit G, Mohapatra S, Sharma S, Deb M. Solitary cysticercosis affecting deltoid muscle: A rare entity. Ann Trop Med Public Health 2015; 8: 210-1.

[11] Ramraje S., Bhatia V., Goel A. Solitary intramuscular cysticercosis - a report of two cases. Australas Med J. 2011; 4 (1): 58-60.

[12] Holzapfel B. M., Schaeffeler C., Banke I. J., Waldt S. A $37-$ year-old man with a painless growing mass of the thorax. Clin Orthop Relat Res. 2010; 468 (4): 1193-1198.

[13] Rangdal SS, Prabhakar S, Dhatt S S, Prakash M, Dhillon MS. Isolated Muscular Cysticercosis: A RarePseudotumor and Diagnostic Challenge, can It be treated Nonoperatively? A Report of Two Cases and Review of Literature. J Postgrad Med Edu Res 2012; 46 (1): 43-48.

[14] Singal R, Mittal A, Gupta S, Gupta R, Sahu P, Gupta A. Intramuscular cysticercosis diagnosed on ultrasonography in thigh: A rare case report. North Am J Med Sci 2010; 2: 162-4.

[15] Singh RP. Intramuscular cysticercosis: The solitary reaper. Ann Afr Med 2014; 13: 53-4.

[16] Asrani A, Morani A. Primary sonographic diagnosis of disseminated muscular cysticercosis. J Ultrasound Med 2004; 23: $1245-8$. 